\title{
Transatlantica
}

Revue d'études américaines. American Studies Journal

1 | 2017

Morphing Bodies: Strategies of Embodiment in

Contemporary US Cultural Practices

\section{American Landscape de Charles Sheeler (1930) : la fumée et l'échelle.}

Jacques Pothier

\section{(2) OpenEdition}

Journals

Édition électronique

URL : https://journals.openedition.org/transatlantica/8458

DOI : $10.4000 /$ transatlantica.8458

ISSN : 1765-2766

Éditeur

Association française d'Etudes Américaines (AFEA)

Référence électronique

Jacques Pothier, « American Landscape de Charles Sheeler (1930) : la fumée et l'échelle. »,

Transatlantica [En ligne], 1 | 2017, mis en ligne le 03 décembre 2018, consulté le 20 mai 2021. URL

http://journals.openedition.org/transatlantica/8458; DOI : https://doi.org/10.4000/transatlantica. 8458

Ce document a été généré automatiquement le 20 mai 2021.

\section{(c) (i) () $९$}

Transatlantica - Revue d'études américaines est mise à disposition selon les termes de la licence Creative Commons Attribution - Pas d'Utilisation Commerciale - Pas de Modification 4.0 International. 


\section{American Landscape de Charles Sheeler (1930) : la fumée et l'échelle.}

Jacques Pothier

Charles Sheeler (1883-1965): American Landscape, 1930

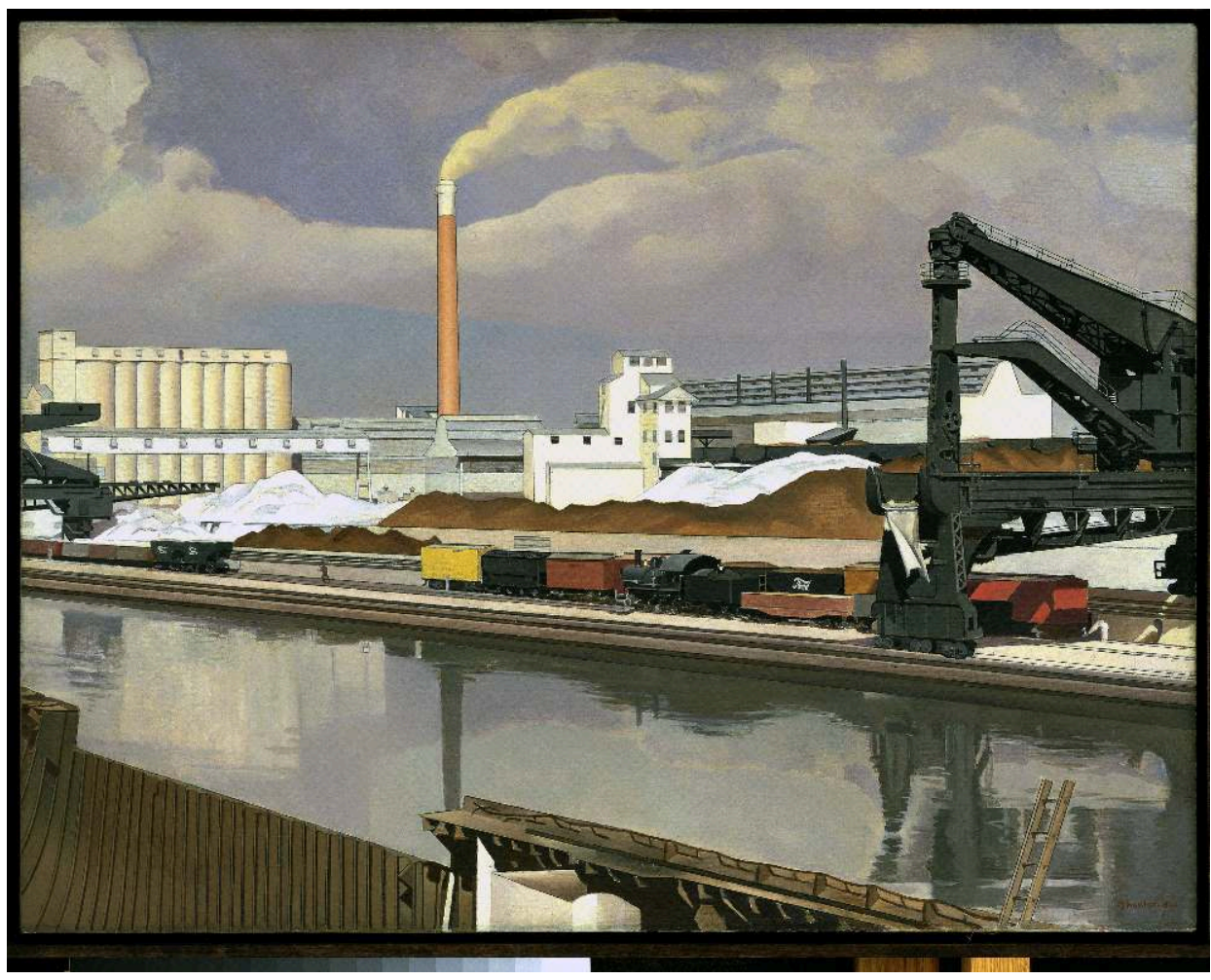

New York, Museum of Modern Art (MoMA). Huile sur toile, 61 x $78.8 \mathrm{~cm}$. Don de Abby Aldrich Rockefeller. Acc. n.: 166.1934

(c) 2018. Image numérique, The Museum of Modern Art, New York/Scala, Florence 
1 American Landscape (1930) est probablement l'œuvre la plus célèbre de Charles Sheeler. Le tableau est à plus d'un titre un classique. Avec The Midnight Ride of Paul Revere de Grant Wood (1931), c'est l'un des deux tableaux de l'exposition La Peinture Américaine des Années Trente: The Age of Anxiety à figurer dans une collection de quarante reproductions grand format qu'en 2008 le National Endowment for the Arts a mise à la disposition des établissements scolaires. Ce projet est accompagné du livret pédagogique Picturing America, disponible en ligne. Au sens où classique signifie « étudié en classe ", l'œuvre la plus célèbre de Charles Sheeler l'est ainsi devenu.

2 Le titre paradoxal du tableau est explicitement générique, en somme conceptuel : il n'interroge pas moins que la célèbre légende Ceci n'est pas une pipe du tableau de Magritte (1928-29), glosée et donc explicitée par le vrai titre La Trahison des images. Ici, le peintre américain laisse son public interpréter la distance entre le titre, American Landscape, et l'horizon d'attente qu'il suscite. Le paysage est historiquement un genre privilégié par les peintres américains, genre qui a même pu les faire apparaître comme attardés à leurs premières visites dans les académies parisiennes passionnées de peintures d'histoire au milieu du XIX ${ }^{\mathrm{ème}}$ siècle, comme l'a raconté Annie Cohen-Solal dans "Un jour, ils auront des peintres" (2000).

Quel dialogue Sheeler instaure-t-il donc avec la grande tradition paysagiste américaine des Thomas Cole, George Inness, Thomas Moran ou Alfred Bierstadt, qui va se prolonger dans les années trente avec les paysages de Grant Wood comme le Fall Plowing (1931) présenté dans l'exposition? Dans la toile de Wood, les fortes ondulations stylisées de la nature sont domestiquées par les stries des sillons, les alignements réguliers des meules ou des arbres, la mosaïque des aplats de couleur unis qui distinguent des parcelles délimitées par des lignes droites. Pas plus de présence humaine visible chez Wood que dans le paysage de Sheeler, apparemment, sauf que c'est chez Sheeler qu'un œil attentif identifiera une minuscule silhouette à égale distante de deux wagons de marchandise sur la voie ferrée. Chez Wood, l'action humaine qui transforme le pays en paysage est évidente, mais c'est en somme la machine qui, au premier plan, s'affirme comme le sujet ordonnateur, en l'absence de l'homme qui devrait la guider: la charrue, comme figée en pleine action (tracer le sillon) par la prise de vue de l'artiste.

4 La peinture est symbolique, presque allégorique, alors que celle de Sheeler s'inscrit dans une froide objectivité. On sait que Charles Sheeler s'est servi pour American Landscape de son travail photographique à l'usine Ford de Detroit, en 1927. Le style « précisionniste » du peintre, inspiré de la prise de vue photographique, l'oppose à la peinture paysagiste en honneur au siècle précédent. Elle s'inscrit dans un récit où l'Amérique tournerait le dos à un idéalisme agrarien jeffersonien pour incarner la modernité industrielle. Les formes géométriques, l'effacement des marques du travail du peintre, contribuent à neutraliser la présence humaine. La taille très réduite du seul sujet peut se lire comme l'effacement de l'être humain dans le triomphe de l'efficacité tayloriste, mais elle rejoint les tableaux de nature des paysagistes du XIX ${ }^{\text {ème }}$ siècle : la quasi disparition de la figure humaine y souligne le sublime. Qu'on pense par exemple à Landscape Scene from the Last of the Mohicans, de Thomas Cole (1827), où un pow-wow indien se déroule dans une clairière entre d'énormes blocs rocheux qui le surplombent, avec dans le lointain une haute montagne impassible qui bouche l'horizon. Sheeler suggère ainsi que le paysage industriel américain contemporain n'est pas en rupture, mais en continuité avec le paysagisme du siècle précédent : il rejoint le sublime de la 
wilderness américaine. D'un siècle à l'autre, le sublime est ainsi une constante qui authentifie l'américanité préservée du paysage.

5 Le paysage pastoral célébrait l'équilibre harmonieux entre nature et civilisation. Equilibre fragile, presque utopique, fréquemment menacé par une temporalité tragique: ce paradigme est au cœur du célèbre tableau de Nicolas Poussin, Les bergers d'Arcadie (1639). Le memento mori, l'intrusion de la mort dans un paysage idyllique se matérialise par la vue d'un serpent dans le Paysage avec Orphée et Eurydice (1650-1653) autre tableau célèbre de Poussin. Leo Marx a montré dans The Machine in the Garden que la version américaine de ce memento mori apparaît souvent comme l'intrusion de la locomotive dont le sifflet et la fumée s'imposent soudainement dans le paysage ; ainsi dans The Lackawanna Valley de George Inness (1855), le train tiré par sa locomotive à vapeur paraît pénétrer en éclaireur le paysage pastoral du premier plan, alors que des usines à l'arrière-plan en présagent l'avenir industriel. Plus optimiste encore, le tableau allégorique de John Gast, American Progress (1872), fait du train à vapeur l'agent d'un progrès qui dissipe les obscurités du monde pré-industriel, fidèle à l'esprit de la «Destinée manifeste». Leo Marx souligne l'ambiguïté de cette pénétration: memento mori de l'âge pastoral jeffersonien, la machine à vapeur vient parachever l'identité américaine du paysage. Alors que dans l'ancien monde l'industrialisation entraîne les malheurs de la vie urbaine, l'optimisme américain permet d'espérer un progrès heureux, où la technologie créerait une forme d'Arcadie post-pastorale, réconciliant nature et civilisation industrielle.

6 C'est en somme l'aboutissement de ce rêve américain que montre Sheeler. La comparaison avec la composition du paysage de Poussin montre que le paysage américain a bien conservé les éléments essentiels du paysage classique, et d'abord une étendue d'eau et le ciel. L'eau chez Sheeler n'est ni un lac, ni la mer, ni un cours d'eau comme chez Poussin, mais un canal rectiligne, comme tiré à la règle, sans flux apparent (mais le Tibre du Paysage avec Orphée et Eurydice n'est pas plus animé chez Poussin, bien que des baigneurs s'y ébattent). Chez Poussin comme chez Sheeler, le plan d'eau réfléchit le paysage, mais il le fait à peine : les bâtisses blanches du centre du tableau de Sheeler se dérobent à la réflexion, comme le fait chez Poussin la silhouette du Château Saint-Ange, d'où s'échappent deux épaisses colonnes de fumée noire qui se mêlent aux nuages également menaçants, annonciateurs de tragédie. Chez Sheeler, la fumée abondante qui s'échappe de la cheminée d'usine semble tout simplement produire les nuages, comme une gracile brosse de peintre, ou plutôt un pistolet à peinture industriel. Le convoi du train à vapeur est arrêté en travers du paysage, la fumée de sa locomotive transposée sans doute en fumée d'usine. Les grands arbres, bergeries, fabriques et moulins de la tradition pastorale sont remplacés par un pont roulant, des silos, hangars et autres bâtiments industriels. Au centre, un bâtiment blanc avec des fenêtres est le seul indice d'une habitation humaine. Il surplombe la scène, comme les châteaux italiens dans les paysages mythologiques de Poussin. Juste en dessous de la cheminée, un wagon vivement coloré, en contraste avec les teintes sombres du reste des wagons de marchandise, rappelle le pan de mur jaune de la Vue de Delft par Vermeer (1660), et le paysage empli de nuages se fait alors flamand.

7 Même dans le plus paisible des paysages pastoraux de Poussin, l'orage menace. Ainsi, Paysage par temps calme (1651), au titre on ne peut plus notionnel, est une pastorale un peu froide, sans référence mythologique, où la sérénité des eaux trouve un écho dans le défilement horizontal des troupeaux et la douceur poudrée de la lumière. Pourtant déjà 
les nuages s'animent au-dessus du château, tandis qu'à gauche, devant la bergerie, un cavalier s'élance au galop pour sortir du champ, et surtout qu'un feu gigantesque apparaît à la droite du château'. L'effet chez Sheeler est comparable: comme l'a remarqué Leo Marx, il donne à son paysage industriel une impression d'ordre, de paix et d'harmonie qui rappelle les codes de la pastorale (Marx 355-6). Les wagons du train semblent être à l'arrêt, aucune activité n'est captée sous le pont roulant, aucune péniche sur le canal. Seule la cheminée semble en action. L'industrialisation ne suggère pas de tension, de vitesse, ni de violence, mais plutôt un équilibre apaisé. Mais la nature a disparu du paysage. Le seul élément d'un monde pastoral effacé consiste en un bout d'échelle de bois au premier plan dans le coin droit, qui semble ne mener nulle part, mais renvoie sans doute à une activité professionnelle traditionnelle, faisant ainsi figure de vestige d'un mode de vie suranné. Peut-être faut-il y voir, comme l'a suggéré Michael Sacasas dans «The Smartphone in the Garden » (2012), la reprise discrète par Sheeler du motif du memento mori de la peinture de paysage pastorale ?

Tout en célébrant le triomphe de l'industrie, de la modernité, et la disparition d'une nature sauvage, Sheeler rend hommage aux paysagistes et en réhabilite l'esthétique. Les éléments traditionnels de la peinture de paysage (plans d'eau, montagnes, bâtisses, arbres) se réincarnent en canal artificiel, silos, usines, cheminées et machines. Alors que le paysage anglais joue à s'effacer pour retrouver une harmonie naturelle, le paysage américain se construit comme conquête de l'ordre et de l'harmonie, dont la nature est effacée. Dès l'officialisation en 1864 du mouvement de protection de la vallée de Yosemite par Abraham Lincoln, qui est confiée à l'Etat de Californie ${ }^{2}$, le sublime espace naturel américain appartient au domaine de la "récréation " des classes qui peuvent s'en offrir le loisir.

Décrivant les déserts du sud-ouest à la même époque dans Death Comes For the Archbishop, (1927), Willa Cather soulignait que pour son personnage qui découvrait ces contrées, la nature était encore sauvage, exempte d'empreinte humaine : « The country was still waiting to be made into a landscape.» (Cather 95). Wood et Sheeler témoignent de la "fabrique » du pays. Sheeler répond à Wood : ce n'est pas l'idéal jeffersonien du quadrillage de l'espace en "hundreds " saxons qui a été le modèle américain, mais celui de l'industrialisation ${ }^{3}$. La machine n'a pas seulement structuré le jardin, elle l'a finalement subrepticement supplanté.

\section{BIBLIOGRAPHIE}

Cather, Willa. Death Comes For the Archbishop. NY: Knopf, 1927; Vintage, 1971.

Cohen-Solal, Annie. "Un jour, ils auront des peintres". Paris, Gallimard, 2000.

Cronon, William. "The Trouble with Wilderness; or, Getting Back to the Wrong Nature" in William Cronon, ed., Uncommon Ground: Rethinking the Human Place in Nature, New York: W. W. Norton \& Co., 1995, 69-90 
Marx, Leo. The Machine in the Garden : Technology and the Pastoral Ideal in America. London: Oxford University Press, 1964, 1981.

Merrill, Linda; Lisa Rogers; Kaye Passmore, Picturing America: Teachers Resource Book. Pilot Resource Book (4.29 MB). Washington DC: National Endowment for the Arts, 2008.

Sacasas Michael. . The Smartphone in the Garden ». L.M. Sacasas. N.p., 23 juillet 2012. Web. 26 févr. 2017.

---. « The Smart Phone [sic] in the Garden, Part Two ». L.M. Sacasas. N.p., 24 juillet 2012. Web. 26 févr. 2017

\section{Iconographie}

Cole, Thomas. Landscape Scene from The Last of the Mohicans, huile sur toile, $83,8 \mathrm{~cm} \mathrm{x} 109 \mathrm{~cm}, 1827$, Cooperstown, NY: Fenimore Art Museum. https://www.wikiart.org/en/thomas-cole/scene-fromthe-last-of-the-mohicans-by-james-fenimore-cooper-1827

Inness, George. The Lackawanna Valley, huile sur toile, 86 x $127.5 \mathrm{~cm}, \mathrm{c.}$ 1856. Washington D.C., National Gallery of Art. https://commons.wikimedia.org/wiki/File\%3AGeorge_Inness_-

_The_Lackawanna_Valley___Google_Art_Project.jpg

Poussin, Nicolas. Les bergers d'Arcadie, huile sur toile, 85 x $121 \mathrm{~cm}$, c. 1638. Musée du Louvre. https://commons.wikimedia.org/wiki/File\%3ANicolas_Poussin_-

_Et_in_Arcadia_ego_(deuxi\%C3\%A8me_version).jpg

---. Paysage avec Orphée et Eurydice, huile sur toile, 124 x 200 cm, c. 1648. Musée du Louvre.

https://commons.wikimedia.org/wiki/

File\%3APaysage_avec_Orph\%C3\%A9e_et_Eurydice_\%E2\%80\%93_Nicolas_Poussin_\%E2\%80\%93_Mus\%C3\%A9e_du_Louvre\%2C_INV_7307

---. Paysage par temps calme [Landscape with a Calm], huile sur toile, 97 x $131 \mathrm{~cm}, 1650-1651$.

Getty Center. https://commons.wikimedia.org/wiki/File:Nicolas_Poussin_(French_-

_Landscape_with_a_Calm_-_Google_Art_Project.jpg

Sheeler, Charles. American Landscape, huile sur toile, 61 x 79 cm, 1930. New York: MoMA In

Merrill, Linda; Lisa Rogers; Kaye Passmore, Picturing America, 2008.

Vermeer, Johannes, Vue de Delft (Gezicht op Delft). huile sur toile, c. 1660, La Haye : Mauristhuis https://fr.wikipedia.org/wiki/Vue_de_Delft

Wood, Grant. Fall Plowing, huile sur toile, 76,2 x 103,5 cm, 1931. Moline, Illinois: John Deere Art Collection https://www.wikiart.org/en/grant-wood/fall-plowing-1931

\section{NOTES}

1. Détail signalé par Romy Sutherland (UCLA), ce dont je la remercie.

2. An Act Authorizing a grant to the State of California of the "Yo-Semite Valley, " and of the land embracing the «Mariposa Big Tree Grove ». 30 juin 1864.

3. Plusieurs colonies britanniques avaient repris le terme hundred pour désigner une division administrative territoriale. Lors du débat pour la «Land Ordinance » (1785), attaché à l'héritage saxon auquel l'étymologie du terme se rattache, Thomas Jefferson avait suggéré de découper les territoires publics fédéraux en hundreds carrés de dix miles de côté. La proposition ne fut pas retenue. 


\section{AUTEUR}

JACQUES POTHIER

Université Versailles St Quentin 